\title{
Power System Transient Stability Assessment Based on PCA and Support Vector Machine
}

\author{
Jingxuan Tang ${ }^{a}$, Huibin Sui ${ }^{b}$ \\ College of Electrical Engineering, Shandong University, Shandong 250061, China. \\ a Corresponding author: tjxaihg@163.com \\ bsuihuibin@sdu.edu.cn
}

\begin{abstract}
Keywords: transient stability; principal component analysis; feature reduction dimension; support
\end{abstract} vector machine.

\begin{abstract}
Combining the synchronized phasor measurement unit (PMU), a power system transient stability assessment method based on principal component analysis and support vector machine is proposed. Firstly, the PMU data is obtained through simulation and the original feature set is constructed. Then the principal feature analysis (PCA) is used to compress the original feature set and reduce the feature size. The obtained main components contain sufficient information of the initial sample and are used as input to Support Vector Machine (SVM) to train and test the sample. The classification effect of New England 10-machine 39-bus system is analyzed. The results show that the proposed model is accurate and effective for power system transient stability analysis.
\end{abstract}

\section{Introduction}

With the continuous development of the national economy and society, the requirements for the safe and stable operation of the power system and the reliability of power supply are also increasing. At present, the electric power system reform in China is continuously deepening and the power system is developing towards long distances and UHV. Various types of large-scale energy storage components, electric car charging piles and other new types of loads are continuously connected, and the cross-regional large-capacity tie line transmission system gradually put into operation, making the power system operation closer to the stability limit. The stability analysis and dispatching operation of the power system is facing severe challenges.

Traditional transient stability assessment methods include domain simulation method, direct method and extended equal area method. The time-domain simulation method is slow in calculation and cannot provide stability margin, so it is difficult to apply to real-time online analysis. Direct method and extended equal area method can obtain the stability margin of the system, but it is limited to the application under the complex model, and cannot fully meet the requirements of online calculation.

With the maturity of wide-area measurement technology and the development of big data theory, machine learning has become one of the main methods for on-line stability assessment of power systems with advantages such as high accuracy, short time-consuming, and low-dimension. Support vector machine (SVM) algorithm has been widely used due to its powerful generalization ability, which provides a way for off-line training and online analysis of transient stability assessment. In order to reduce the dimension of the feature to input to SVM, the main component analysis method that can integrate most of the original feature information is used to compress the data.

\section{Principal Component Analysis}

Principal component analysis (PCA) is a commonly used dimension reduction method in machine learning. Its basic idea is to replace a large number of related variables with a set of few independent variables, while retaining the information of original variables as much as possible. The new variable obtained is called the principal component, and its model is shown in Fig.1. From statistical point of 
view, variance can be used to represent the amount of information contained in a variable. The larger the variance, the greater the amount of information that the principal component contains.

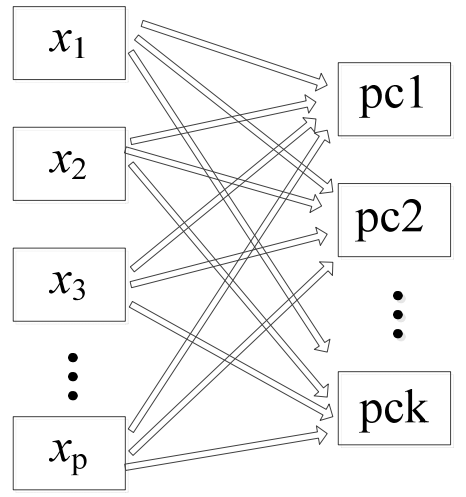

Figure 1. Principal component analysis model

Let $X=\left(x_{1}, x_{2}, x_{3}, \cdots, x_{p}\right)$ be a $\mathrm{p}$-dimensional random vector and linearly combine the original $\mathrm{p}$ indicators to obtain new $\mathrm{k}$ comprehensive indicators, which is denoted as the main component $\mathrm{Y}(\mathrm{i}=1$, $2, \ldots \mathrm{k} ; \mathrm{k} \leq \mathrm{p}$ ), that is:

$$
\left\{\begin{array}{c}
y_{1}=w_{11} x_{1}+w_{12} x_{2}+\cdots w_{1 p} x_{p} \\
y_{2}=w_{21} x_{1}+w_{22} x_{2}+\cdots w_{2 p} x_{p} \\
\vdots \\
y_{2}=w_{p 1} x_{1}+w_{p 2} x_{2}+\cdots w_{p p} x_{p}
\end{array}\right.
$$

Among them, the wij coefficient needs to satisfy:

(1) Each variable y1, y2, .., yk is mutually independent.

(2) $\mathrm{W}$ is an orthogonal matrix, and $w_{k 1}^{2}+w_{k 2}^{2}+w_{k 3}^{2}+\cdots+w_{k p}^{2}=1$.

(3) The variance of the variable y1 is greater than or equal to the variance of $y 2$, and the variance of the variable is successively decreasing.

When the above three conditions are satisfied, the $\mathrm{Y}$ components $\mathrm{y} 1, \mathrm{y} 2, \cdots$, yk which obtained after the conversion are the first, second, ..., n-th principal components of $\mathrm{X}$, respectively.

The degree that the main component presents the original information amount can be represented by the contribution rate and the cumulative contribution rate. Let $\mathrm{C}$ be the covariance matrix of the random vector $X$, whose eigenvalues are $\lambda 1, \lambda 2, \cdots, \lambda p$, and $\lambda 1 \geqslant \lambda 2 \geqslant \cdots \geqslant \lambda p \geqslant 0$. The contribution rate of the $\mathrm{k}$-th principal component is the ratio of the $\mathrm{k}$-th eigenvalue of the covariance matrix to the sum of the eigenvalues. The larger the ratio, the more information that the principal component presents. Its calculation formula is:

$$
\omega_{i}=\lambda_{k} / \sum_{i=1}^{p} \lambda_{i}
$$

The cumulative contribution of the k-th principal component is the ratio of the sum of the first $\mathrm{k}$ eigenvalues to the sum of the whole eigenvalues of the covariance matrix. The larger the ratio, the more information of the original data that the first $\mathrm{k}$ principal components represent. Its calculation formula is:

$$
\beta_{i}=\sum_{i=1}^{k} \lambda_{i} / \sum_{i=1}^{p} \lambda_{i}
$$

In practical applications, the first $\mathrm{k}$ principal components are usually selected so that the cumulative contribution rate meets a certain threshold, and $\mathrm{k}$ primary components are used in place of the original $\mathrm{p}$ variable indicators for subsequent data analysis to achieve the purpose of data compression and dimensionality reduction, while also extracting the main features of the data.

\section{Support Vector Machine}

Support vector machine (SVM) is a new machine learning method based on statistical learning theory that put forward by Vapnik et al. It has significant advantages in solving small sample, 
nonlinear and high dimensional pattern recognition. The main idea of support vector machine classification method is to find an optimal hyperplane in high dimensional space as the segmentation surface of the sample, and to maximize the classification interval.

The SVM classification problem can be described as: solve the optimal classification hyperplane when given a set of training sets $S=\left\{\left(x_{i}, y_{i}\right) \mid x_{i} \in R^{n}, y_{i} \in R\right\}_{i=1}^{l}$. Where xi is the input feature of the $\mathrm{i}$-th description system, yi is the classifier identifier of the $\mathrm{i}$-th sample, and 1 is the sample number. When the training set is linearly separable, the optimal classification function $f(x)=\operatorname{sgn}[(w \cdot x)+b]$ is determined by solving the following optimization problems.

$$
\left\{\begin{array}{l}
\min \frac{1}{2} w^{T} w+C \sum_{i=1}^{l} \xi_{i} \\
\text { s.t } y_{i}\left[\left(w^{T} \cdot x_{i}\right)+b\right] \geq 1-\xi_{i} \\
\xi_{i} \geq 0 \quad \forall i=1,2, \cdots, l
\end{array}\right.
$$

In the formula, $\mathrm{C}>0$ is the penalty factor, and $\xi \mathrm{i}$ is the slack variable.

Introduce the Lagrange multipliers $\alpha \mathrm{i}, \gamma \mathrm{i}$ and construct the Lagrange function as follows:

$$
\begin{aligned}
& L(w, b, \xi, \alpha, \gamma)=\frac{1}{2} w^{T} w+C \sum_{i=1}^{l} \xi_{i}- \\
& \quad \sum_{i=1}^{l} \alpha_{i}\left\{y_{i}\left[\left(w^{T} \cdot x_{i}\right)+b\right]-1+\xi_{i}\right\}-\sum_{i=1}^{l} \gamma_{i} \xi_{i}
\end{aligned}
$$

This translates into the solution to its dual problem

$$
\left\{\begin{array}{l}
\max \sum_{i=1}^{l} \alpha_{i}-\frac{1}{2} \sum_{i=1, j=1}^{l} y_{i} y_{j} \alpha_{i} \alpha_{j}\left(x_{i}^{T}, x_{j}\right) \\
\text { s.t } 0 \leq \alpha_{i} \leq C, i=1,2, \cdots, l \\
\sum_{i=1}^{l} \alpha_{i} y_{i}=0
\end{array}\right.
$$

Find a set of $\alpha$ that satisfies the above conditions and solve for $\mathrm{w}$ and $\mathrm{b}$.

$$
\begin{gathered}
w=\sum_{i=1}^{l} \alpha_{i} y_{i} x_{i} \\
b=-\frac{\max _{i: y_{i}=-1} w^{T} x_{i}+\min _{i: y_{i}=1} w^{T} x_{i}}{2}
\end{gathered}
$$

When the training set data is linearly inseparable, it can be mapped into a high-dimensional space through a nonlinear mapping map so that it is linearly separable in the high-dimensional space. That is, replace the vector $\mathrm{x}$ with $\psi(\mathrm{x})$ and replace the inner product (xiT,xj) with $K\left(x_{i}, x_{j}\right)=\psi\left(x_{i}\right)^{T} \cdot \psi\left(x_{j}\right)$, which is called the kernel function. The function of $\mathrm{K}(\mathrm{xi}, \mathrm{xj})$ is to input the low-dimensional space vector and calculate the transformed high-dimensional space. The vector inner product value in. In SVM, there are the following four commonly used kernel functions:

$$
\left\{\begin{array}{l}
\text { linear : } K\left(x_{i}, x_{j}\right)=x_{i}^{T} \cdot x_{j} \\
\text { polynomial: } K\left(x_{i}, x_{j}\right)=\left(a x_{i}^{T} x_{j}+b\right)^{d}, a>0 \\
\text { RBF : } K\left(x_{i}, x_{j}\right)=\exp \left(-\gamma\left\|x_{i}-x_{j}\right\|^{2}\right), \gamma>0 \\
\text { sigmoid: } K\left(x_{i}, x_{j}\right)=\tanh \left(a x_{i}^{T} x_{j}+b\right)
\end{array}\right.
$$

\section{Simulation Experiment Analysis}

Simulation experiments are performed in the PSAT simulation software which based on the Matlab platform and python software. Construct sample sets by collecting data based on New England 39 -node system. The fault is set to three-phase short circuit, the system fails at $0.1 \mathrm{~s}$, and the fault is cleared at $0.3 \mathrm{~s}$ or $0.35 \mathrm{~s}$. The load model is set to $80 \%, 90 \%, 100 \%, 110 \%, 120 \%$, and the generator output is changed correspondingly. The simulation time is $4 \mathrm{~s}$. The generator is a 4 th order model and the load is a constant impedance model. The criterion for setting the instability is that the generator's 
maximum work angle difference exceeds $180^{\circ}$, which is denoted by -1 for system instability and 1 for system stability. A total of 390 samples were obtained from simulation, of which 239 were stable and 151 were unstable.

Based on the four characteristics of the generator power angle, angular velocity, output active power, and mechanical power in the sample, a 26-dimensional initial feature set is constructed based on the comprehensive relevant literature. Then, using the principal component analysis method to set the first nine principal components, the cumulative contribution rate at this time has reached more than $95 \%$, and the dimensionality reduction effect is obvious. From the 390 sample set, 273 samples were selected as training samples, of which 168 were stable samples, 105 were unstable samples, and the remaining 117 samples were test samples. Using the support vector machine to identify and classify the samples, the classification accuracy rate reaches 95.72\% (112/117). The results of transient stability assessment show that extracting features by principal component analysis method and using the features to input to the support vector machine can run at a high rate accuracy and feasibility and can be applied online.

\section{References}

[1]. Xue Yusheng. A critical comparison of various methods for transient stability assessment part one: disturbed-measure functions[J]. Automation of Electric Power Systems,2001,25(11):6-11.

[2]. Song Yaqi, Zhou Guoliang, Zhu Yongli. Present status and challenges of big data processing in smart grid[J]. Power System Technology.2013,37(4):927-935.

[3]. Dai Renchang, Zhang Boming, Qi Qihui. Transient stability simulation on artificial intelligence [J]. Proceedings of the CSEE,2002,22(12):1-5.

[4]. Izzri A W N, Mohamed A, Yahya I. A New Method of Transient Stability Assessment in Power Systems Using LS-SVM[C]. Research and Development, 2007.Scored 2007.Student Conference on. IEEE,2008:1-6.

[5]. Wu Qiong, Yang Yihan, Liu Wenying, et al. Electrical power system transient stability on-line prediction based on least squares support vector machine[J]. Proceedings of the CSEE, 2007, 27(25):38-43.

[6]. Ma Qian, Yang Yihan, Liu Wenying, et al. Power system transient stability assessment with combined SVM method mixing multiple input features[J]. Proceedings of the CSEE, 2005,25 (6):17-23.

[7]. Li Guoqing. The study of power system transient stability prediction control[J]. Automation of Electric Power Systems, 1994,18(3):25-32.

[8]. Xu Tao, He Renmu, Wang Peng, et al. Power system transient stability assessment based on statistical learning theory [J]. Proceedings of the CSEE,2003,23(11):51-55.

[9]. Ye Shengyong, Wang Xiaoru, Liu Zhigang, et al. Transient stability assessment based on random forest algorithm[J]. Journal of Southwest Jiaotong University,2008,43(5):573-577.

[10]. Huang Hui, Shu Naiqiu, Li Zipin, et al. Power system transient stability assessment based on information fusion technology[J]. Proceedings of the CSEE,2007,27(16):19-23.

[11]. Li Dahu, Cao Yijia. Power system transient stability analysis based on PMU and hybrid support vector machine[J].Power System Technology,2006,30(9):46-52.

[12]. Jia Rong, Cai Zhenhua, Liu Jing, et al. Power system estimation based on principal component analysis and least square support vector machines. Power System Technology,2006,30(21):7577. 
[13]. Guan Lin, Wang Tongwen, Tang Zongshun. Intelligent algorithm for kernel feature identification and stability-based system division in power grid security monitoring $[\mathrm{J}]$. Automation of Electric Power Systems,2006,30 (21) :22-27.

[14]. Yu Zhihong, Guo Zhizhong. A novel approach for transient stability assessment based on data mining theory[J].Automation of Electric Power Systems, 2003,27(8):45-48.

[15]. Tang Fei, Wang Bo, Zha Xiaoming, et al. Power system transient stability assessment based on two-stage parallel hidden Markov model[J]. Proceedings of the CSEE,2013,33(10):90-97. 